\title{
Symmetries of the Discrete Burgers Equation
}

\author{
R. Hernández Heredero* \\ D. $\operatorname{Levi}^{\dagger}$ \\ P. Winternitz
}

CRM-2568

October 1998

\footnotetext{
*Departamento de Física Teórica II, Facultad de Ciencias Físicas, Universidad Complutense de Madrid, 28040 Madrid, Spain. rafa@ciruelo.fis.ucm.es

†Dipartimento di Fisica "Eduardo Amaldi", Universitá di Roma Tre and INFN, Sezione di Roma Tre, Via della Vasca Navale 84, 00146 Roma, Italy. levi@amaldi.fis.uniroma3.it

${ }^{\ddagger}$ Centre de recherches mathématiques, Université de Montréal, C.P. 6128, succ. Centre-ville, Montréal, QC H3C3J7, Canada. wintern@CRM.UMontreal.CA
} 


\begin{abstract}
A discrete Cole-Hopf transformation is used to derive a discrete Burgers equation that is linearizable to a discrete heat equation. A five-dimensional symmetry algebra is obtained that reduces to the Lie point symmetry algebra of the usual Burgers equation, in the continuous limit. This Lie algebra is used to obtain explicit invariant solutions.
\end{abstract}

\title{
Résumé
}

On utilise une transformation de Cole-Hopf pour obtenir une équation de Burgers discrète qui est linéarisable et peut être ainsi amenée à une équation de la chaleur discrète. Une algèbre de symétrie de dimension cinq est obtenue. Cette algèbre se réduit aux symétries locales de l'équation de Burgers usuelle dans la limite continue. Cette algèbre de symétrie est utilisée pour obtenir des solutions invariantes explicites. 


\section{Introduction}

The purpose of this article is to study a discrete Burgers equation that shares the integrability properties and point symmetries of the continuous Burgers equation.

The Burgers equation [1] is of considerable physical and mathematical interest. On one hand it is widely applicable in hydrodynamics and other areas, since it is the simplest partial differential equation that combines a description of nonlinear effects with that of dissipative ones.

From the mathematical point of view it is the prototype of an equation that is linearizable via a direct coordinate transformation. Indeed, the standard form of the Burgers equation is

$$
u_{t}=u_{x x}+2 u u_{x}
$$

Putting

$$
u=v_{x}
$$

we obtain the potential form of the Burgers equation, namely

$$
v_{t}=v_{x x}+v_{x}^{2}
$$

Finally, setting

$$
w=e^{v}
$$

we obtain the linear heat equation for $w$, namely

$$
w_{t}=w_{x x}
$$

In other words, the standard Burgers equation is transformed into the heat equation by the ColeHopf transformation

$$
u=\frac{w_{x}}{w}
$$

The potential Burgers equation (1.3) has an infinite dimensional Lie algebra of point symmetries that is "inherited" from the linear heat equation [2]. That of the Burgers equation (1.1) is fivedimensional. They both have infinitely many higher symmetries, and allow Bäcklund transformations. Moreover, it is possible to view the linear system

$$
\begin{aligned}
w_{t} & =w_{x x} \\
w_{x} & =u w
\end{aligned}
$$

as a Lax pair for the Burgers equation, i.e. equation (1.1) is the compatibility condition for the pair (1.7).

Our aim is to provide a difference equation in two variables $x$ and $t$ that goes into the standard Burgers equation in the continuous limit and has all of the above properties when considered as a difference equation.

We start out from the discrete linear system consisting of the discrete heat equation and a discrete Cole-Hopf transformation. From it we obtain the required discrete equation for $u(x, t)$ as a compatibility condition.

We then use the known symmetries of the discrete heat equation [3, 4] to derive symmetries of the discrete Burgers equation. The symmetries appear as compatible flows. In the discrete case 
they do not really correspond to point transformations, since they act simultaneously at more than one point of the lattice. In the continuous limit they go into the known point symmetries of the Burgers equation.

This article is part of a research direction devoted to symmetries of difference equations [418]. It is not difficult to discretize linear differential equations while preserving their Lie point symmetries, realized by difference operators, rather than by continuous vector fields [3, 4]. The same cannot be said for nonlinear difference equations. The fact that the Burgers equation is both interesting and linearizable turns it into a most suitable tool for investigating symmetries of nonlinear difference equations. Furthermore, it can serve as a prototype for calculating symmetries of integrable difference equations.

\section{Derivation of the discrete Burgers equation}

\section{$2.1 \quad$ Notations}

We shall user the same conventions and notations as in [4]. Thus, the dependent functions $\phi(x, t)$ and $u(x, t)$ will be continuous functions of continuous variables. The independent variables, $x$ and $t$, while also continuous will be "sampled" at discrete points. Thus, we shall consider a twodimensional rectangular lattice with spacings $\sigma_{x}$ and $\sigma_{t}$ in the $x$ and $t$ directions, respectively. Instead of derivatives, equations will involve variations, or "discrete derivatives" $\Delta_{x}$ and $\Delta_{t}$, and shift operators $T_{x}$ and $T_{t}$. The corresponding definitions are

$$
\begin{array}{rlrl}
T_{x} f(x, t) & =f\left(x+\sigma_{x}, t\right), & & T_{t} f(x, t)=f\left(x, t+\sigma_{t}\right), \\
\Delta_{x} & =\frac{1}{\sigma_{x}}\left(T_{x}-1\right), & \Delta_{t}=\frac{1}{\sigma_{t}}\left(T_{t}-1\right) .
\end{array}
$$

The three quantities $\Delta, T$ and $\sigma$ are not independent, as seen from eq. (2.2). We find sometimes convenient to use all three of them in the same formula, but it must be kept in mind that formulas can be reshuffled using eq. (2.2). Higher discrete derivatives are defined recursively.

\subsection{The discrete Burgers equation as a compatibility condition}

As stated in the Introduction, we first write a discrete linear system, namely

$$
\begin{aligned}
\Delta_{t} \phi & =\Delta_{x x} \phi, \\
\Delta_{x} \phi & =u \phi,
\end{aligned}
$$

consisting of the discrete heat equation and the discrete Cole-Hopf transformation, relating the functions $\phi(x, t)$ and $u(x, t)$.

We first use eq. (2.4) to rewrite eq. (2.3) as

$$
\Delta_{t} \phi=\left[\Delta_{x} u+u T_{x} u\right] \phi
$$

We then require the compatibility of (2.4) and (2.5), i.e. $\Delta_{t} \Delta_{x} \phi=\Delta_{x} \Delta_{t} \phi$ and obtain a condition on $u(x, t)$, which we shall call the "discrete Burgers equation" [19]:

$$
\Delta_{t} u=\frac{1+\sigma_{x} u}{1+\sigma_{t}\left[\Delta_{x} u+u T_{x} u\right]} \Delta_{x}\left(\Delta_{x} u+u T_{x} u\right) .
$$


Eq. (2.6) can be rewritten in many different forms, for instance we can use eq. (2.2) to eliminate all discrete derivatives in terms of shift operators $T$ and spacings $\sigma$.

The continuous limit of all the discrete equations is obtained by taking $\sigma_{x} \rightarrow 0, \sigma_{t} \rightarrow 0$. We have

$$
\Delta_{t} \rightarrow \frac{\partial}{\partial t}, \quad T_{t} \rightarrow 1, \quad \sigma_{t} \rightarrow 0
$$

and similarly for $\Delta_{x}, T_{x}$ and $\sigma_{x}$. In the continuous limit eq. (2.6) goes into the usual Burgers equation in the form (1.1).

We mention that a related discrete Burgers equation appeared in a different context in ref. [19], and that an "ultradiscrete" version of it found an application to traffic flow modeling in [20]. A semidiscrete Burgers equation (with continuous time $t$ ) was also introduced earlier and its integrability was analyzed [21]. It can be reobtained here by taking the limit $\sigma_{t} \rightarrow 0$ for $\sigma_{x}$ fixed, $\sigma_{x} \neq 0$. Using the Lax equation (2.4) we can derive a Bäcklund transformation for the discrete Burgers equation, in the same way as was done in the semidiscrete case [21]. The Bäcklund transformation relates a solution $u(x, t)$ of eq. (2.6) to a new solution $\tilde{u}(x, t)$ of the same equation:

$$
\tilde{u}=\frac{p u+\left(T_{x} u\right)\left(1+\sigma_{x} u\right)}{p+1+\sigma_{x} u},
$$

where $p$ is an arbitrary constant.

\section{Symmetries of the discrete Burgers equation}

\subsection{General procedure}

To obtain the symmetries of the discrete Burgers equation we proceed with the same strategy as used for deriving the equation itself. We start from the symmetries of the discrete heat equation [3, 4], require their compatibility with the Cole-Hopf transformation (2.4) and express the result in terms of the function $u(x, t)$.

As in ref. [4] we make use of an evolutionary formalism for difference equations. The symmetry algebra for a difference equation involving one dependent variable and two independent variables $(x$ and $t)$ will be realized by vector fields of the form

$$
\hat{X}=Q\left(x, t, T_{x}^{a} T_{t}^{b} u, T_{x}^{c} T_{t}^{d} \Delta_{x} u, T_{x}^{e} T_{t}^{f} \Delta_{t} u, \ldots\right) \partial_{u},
$$

where $T_{x}^{a} T_{t}^{b}$ indicates that arbitrary shifts in $x$ and $t$ may be present. For linear difference equations a rather restrictive Ansatz on the form of the function $Q$ provides symmetries that reproduce all point symmetries in the continuous limit. The Ansatz is [4]:

$$
Q=\sum_{c, d} \xi(x, t) T_{x}^{c} T_{t}^{d} \Delta_{x} u+\sum_{e, f} \tau(x, t) T_{x}^{e} T_{t}^{f} \Delta_{t} u-\sum_{a, b} \psi(x, t) T_{x}^{a} T_{t}^{b} u
$$

Each element of the symmetry algebra provides a flow that is compatible with the studied equation, namely

$$
u_{\lambda}=Q
$$

where $\lambda$ is a group parameter that can also be viewed as a new "time". Thus, both for differential and difference equations the existence of symmetries is tantamount to the existence of commuting flows. 
For the heat equation (2.1) the elements of the symmetry algebra have the form (3.2). More specifically, an infinite-dimensional Lie algebra was obtained in ref. [4], involving arbitrary powers of shift operators. A six-dimensional subalgebra was identified that is isomorphic to the symmetry algebra of the continuous heat equation. Both in the continuous and discrete case, we factor out an infinite-dimensional ideal, corresponding to the linear superposition principle. In terms of commuting flows (see eq. (3.3)) we write a basis for this finite-dimensional Lie algebra as

$$
\begin{aligned}
\phi_{\lambda_{1}}= & \Delta_{t} \phi, \\
\phi_{\lambda_{2}}= & \Delta_{x} \phi, \\
\phi_{\lambda_{3}}= & 2 t T_{t}^{-1} \Delta_{x} \phi+x T_{x}^{-1} \phi+\frac{1}{2} \sigma_{x} T_{x}^{-1} \phi, \\
\phi_{\lambda_{4}}= & 2 t T_{t}^{-1} \Delta_{t} \phi+x T_{x}^{-1} \Delta_{x} \phi+\left(1-\frac{1}{2} T_{x}^{-1}\right) \phi, \\
\phi_{\lambda_{5}}= & t^{2} T_{t}^{-2} \Delta_{t} \phi+t x T_{t}^{-1} T_{x}^{-1} \Delta_{x} \phi+\frac{1}{4} x^{2} T_{x}^{-2} \phi \\
& \quad+t\left(T_{t}^{-2}-\frac{1}{2} T_{t}^{-1} T_{x}^{-1}\right) \phi-\frac{1}{16} \sigma_{x}^{2} T_{x}^{-2} \phi, \\
\phi_{\lambda_{6}}= & \phi .
\end{aligned}
$$

In the continuous limit $(3.4), \ldots,(3.9)$ go into the usual generators of time translations, space translations, Galilei transformations, dilations, projective transformations and the multiplication by a constant, respectively.

Each of these symmetries can be acted upon by arbitrary functions of the shift operators $f\left(T_{x}, T_{t}\right)$, obtaining further symmetries. In the continuous limit we have $f\left(T_{x}, T_{t}\right)=f(1,1)=$ const, so all these "higher" symmetries reduce to the six original ones for $\sigma_{x} \rightarrow 0, \sigma_{t} \rightarrow 0$. Among these symmetries two are particularly relevant, namely

$$
\begin{aligned}
\phi_{\lambda} & =\phi_{t}, \\
\phi_{\lambda} & =\phi_{x} .
\end{aligned}
$$

These are the usual $t$ and $x$ translations, that can be obtained using the well known formula

$$
\phi_{z}=\sum_{k=0}^{\infty} \frac{(-1)^{k}}{k+1}\left(T_{z}-1\right)^{k} \Delta_{z} \phi
$$

(with $z=x$, or $z=t$ ).

\subsection{Symmetries and the Cole-Hopf transformation}

All symmetries of the heat equation can be written symbolically as

$$
\phi_{\lambda}=\mathcal{S} \phi
$$

where $\mathcal{S}=\mathcal{S}\left(x, t, \phi, T_{x}, T_{t}, \Delta_{x}, \Delta_{t}, \partial_{x}, \partial_{t}\right)$ is a linear operator that can in each case be read off from eq. $(3.4), \ldots,(3.11)$.

We use the Cole-Hopf transformation (2.4) to transform symmetries of the heat equation (2.3) into those of the discrete Burgers equation (2.6). Let us first prove a general result.

Theorem Let eq. (3.13) represent a symmetry of the discrete heat equation (2.3). Then the same operator $\mathcal{S}$ provides a symmetry of the discrete Burgers equation (2.6) via the formula

$$
u_{\lambda}=\left(1+\sigma_{x} u\right) \Delta_{x}\left(\frac{\mathcal{S} \phi}{\phi}\right)
$$


where $\mathcal{S} \phi / \phi$ can be (and must be) expressed entirely in terms of $u(x, t)$, its variations and their shifted values.

Proof: Let us require that the symmetry (3.13) and the Cole-Hopf transformation (2.4) be compatible. Equality of cross-derivatives

$$
\frac{\partial}{\partial \lambda}\left(\Delta_{x} \phi\right)=\Delta_{x} \phi_{\lambda}
$$

implies

$$
u_{\lambda}=\frac{\Delta_{x}(\mathcal{S} \phi)-u \mathcal{S} \phi}{\phi}
$$

On the other hand, a direct calculation yields

$$
\begin{aligned}
\Delta_{x}\left(\frac{\mathcal{S} \phi}{\phi}\right) & =\frac{1}{\sigma_{x}}\left[\frac{T_{x}(\mathcal{S} \phi)}{T_{x} \phi}-\frac{\mathcal{S} \phi}{\phi}\right] \\
& =\frac{1}{\sigma_{x}\left(T_{x} \phi\right) \phi}\left\{\phi\left[T_{x}(\mathcal{S} \phi)-\mathcal{S} \phi\right]-\left[\left(T_{x} \phi\right)(\mathcal{S} \phi)-\phi(\mathcal{S} \phi)\right]\right\}, \\
\Delta_{x}\left(\frac{\mathcal{S} \phi}{\phi}\right) & =\frac{1}{T_{x} \phi}\left[\Delta_{x}(\mathcal{S} \phi)-u(\mathcal{S} \phi)\right] .
\end{aligned}
$$

Multiplying by $\sigma_{x} u$ and using the Cole-Hopf transformation again we obtain

$$
\sigma_{x} u \Delta_{x}\left(\frac{\mathcal{S} \phi}{\phi}\right)=\frac{\Delta_{x}(\mathcal{S} \phi)-u(\mathcal{S} \phi)}{\phi}-\Delta_{x}\left(\frac{\mathcal{S} \phi}{\phi}\right) .
$$

Using (3.16), we replace the first term on the right hand side of (3.19) by $u_{\lambda}$, and obtain eq. (3.14).

In order to show that the fraction $\mathcal{S} \phi / \phi$ can be expressed in terms of the function $u$, it is sufficient to write $\Delta_{t} \phi, \Delta_{x} \phi, T_{x} \phi, T_{t} \phi, T_{x}^{-1} \phi$, etc, as expressions depending on $u$, times $\phi$ (see eq. (3.4), . , (3.9)). The necessary formulas are obtained from eq. (2.4) and (2.5), namely

$$
\begin{array}{cc}
\Delta_{x} \phi=\phi u, & \Delta_{t} \phi=\phi v, \\
T_{x} \phi=\phi\left(1+\sigma_{x} u\right), & T_{t} \phi=\phi\left(1+\sigma_{t} v\right), \\
T_{x}^{-1} \phi=\phi T_{x}^{-1} \frac{1}{1+\sigma_{x} u}, & T_{t}^{-1} \phi=\phi T_{t}^{-1} \frac{1}{1+\sigma_{t} v},
\end{array}
$$

where we have introduced the notation

$$
v=\Delta_{x} u+u T_{x} u
$$

Applying the Theorem to the one-dimensional subalgebras $\phi_{\lambda_{1}}, \ldots, \phi_{\lambda_{6}}$ of eq. (3.4), ., (3.9), we obtain the corresponding symmetries of the discrete Burgers equation. A basis for this Lie algebra 
is given by the following flows:

$$
\begin{aligned}
u_{\lambda_{1}}= & \left(1+\sigma_{t} v\right) \Delta_{t} u \\
u_{\lambda_{2}}= & \left(1+\sigma_{x} u\right) \Delta_{x} u \\
u_{\lambda_{3}}= & \left(1+\sigma_{x} u\right) \Delta_{x}\left[2 t T_{t}^{-1} \frac{u}{1+\sigma_{t} v}+\left(x+\frac{1}{2} \sigma_{x}\right) T_{x}^{-1} \frac{1}{1+\sigma_{x} u}\right], \\
u_{\lambda_{4}}= & \left(1+\sigma_{x} u\right) \Delta_{x}\left[2 t T_{t}^{-1} \frac{v}{1+\sigma_{t} v}+x T_{x}^{-1} \frac{u}{1+\sigma_{x} u}-\frac{1}{2} T_{x}^{-1} \frac{1}{1+\sigma_{x} u}\right], \\
u_{\lambda_{5}}= & \left(1+\sigma_{x} u\right) \Delta_{x}\left[t^{2} T_{t}^{-1}\left(\frac{1}{1+\sigma_{t} v} T_{t}^{-1} \frac{v}{1+\sigma_{t} v}\right)\right. \\
& +t x T_{x}^{-1}\left(\frac{1}{1+\sigma_{x} u} T_{t}^{-1} \frac{u}{1+\sigma_{t} v}\right)+\frac{1}{4}\left(x^{2}-\frac{\sigma_{x}^{2}}{4}\right) T_{x}^{-1}\left(\frac{1}{1+\sigma_{x} u} .\right. \\
& \left.\quad T_{x}^{-1} \frac{1}{1+\sigma_{x} u}\right)+t T_{t}^{-1}\left(\frac{1}{1+\sigma_{t} v} T_{t}^{-1} \frac{1}{1+\sigma_{t} v}\right) \\
& \left.-\frac{1}{2} t T_{x}^{-1}\left(\frac{1}{1+\sigma_{x} u} T_{t}^{-1} \frac{1}{1+\sigma_{t} v}\right)\right] \\
u_{\lambda_{6}}= & 0 .
\end{aligned}
$$

The quantity $v$ is defined in eq. (3.21).

Thus, the six-dimensional symmetry algebra of the discrete heat equation gives rise to a fivedimensional symmetry algebra of the discrete Burgers equation. The same is true in the continuous case.

The fact that the flows $(3.22), \ldots,(3.26)$ commute with the flow of the discrete Burgers equation (2.6) was also checked directly on a computer (using Mathematica).

In the continuous limit, eq. (3.22), . , (3.26) go over correctly into the well known symmetries of the usual Burgers equation (1.1), namely time translations, space translations, Galilei boosts, dilations and projective transformations. The commutation relations in the discrete case are the same as in the continuous one.

We can show directly that the usual space and time translations are also symmetries of the discrete Burgers equation:

$$
\begin{aligned}
u_{\lambda_{t}} & =u_{t}, \\
u_{\lambda_{x}} & =u_{x} .
\end{aligned}
$$

Indeed, it is easy to check that the corresponding $\lambda$-flows commute with the $t$-flow given by eq. (2.6).

Further symmetries can be generated from other symmetries of the heat equation. Thus, the symmetry (3.9) of the discrete heath equation does not provide a symmetry of the discrete Burgers equation (see eq. (3.27)). The "higher" symmetries of the heat equation given by $\phi_{\mu}=T_{x}^{a} \phi$ will give new symmetries. Indeed, we have for instance for $a=-1$

$$
u_{\mu}=\left(1+\sigma_{x} u\right) \Delta_{x}\left(\frac{T_{x}^{-1} \phi}{\phi}\right)
$$

i.e.

$$
u_{\mu}=\left(1+\sigma_{x} u\right) T_{x}^{-1} \Delta_{x}\left(\frac{1}{1+\sigma_{x} u}\right) .
$$

In the continuous limit this symmetry goes into $u_{\mu}=0$, i.e. it becomes trivial. 


\section{Symmetry reduction for the discrete Burgers equation}

\subsection{General comments}

For a partial differential equation, symmetry reduction is a means for decreasing the number of independent variables in the equation. One way of viewing it is that one looks for fixed points of the $\lambda$-flow corresponding to a certain symmetry. Thus we have the so called surface condition

$$
u_{\lambda}=Q\left(x, t, u, u_{x}, u_{t}, \ldots\right)=0
$$

Eq. (4.1) is to be solved in conjunction with the equation under consideration. Often one can solve eq. (4.1) directly, in particular using the method of characteristics, if it is a first order linear or quasilinear equation. The result is then substituted into the original equation and this leads to a reduction. Alternatively, eq. (4.1) can be used to eliminate all $t$-derivatives (or $x$-derivatives) from the equation, thus reducing it to an ordinary differential equation (with the other variable as a parameter).

Solutions obtained in this manner are called group invariant solutions [2]. They form a very important class of explicit solutions of nonlinear partial differential equations.

If eq. (4.1) is implemented, together with the partial differential equation to be solved, the resulting system may have some further symmetries, inherited from the symmetry algebra $\mathcal{L}$ of the original equation. More specifically, let $X_{e}=Q \partial_{u}$ be the corresponding evolutionary vector field. The symmetries that will survive, once eq. (4.1) is imposed, form a subalgebra $\mathcal{L}_{0} \subset \mathcal{L}$, where $\mathcal{L}_{0}$ is the normalizer algebra of $X_{e}$ :

$$
\mathcal{L}_{0}=\left\{Y_{e} \subset \mathcal{L} \mid\left[Y_{e}, X_{e}\right]=\lambda X_{e}\right\}, \quad \lambda \in \mathbb{R} .
$$

Let us illustrate the situation using the continuous Burgers equation as an example. The symmetry algebra $\mathcal{L}$ in the usual vector field formalism has a basis given by

$$
\begin{gathered}
P_{0}=\partial_{t}, \quad P_{1}=\partial_{x}, \quad B=t \partial_{x}-\frac{1}{2} \partial_{u}, \quad D=2 t \partial_{t}+x \partial_{x}-u \partial_{u} \\
R=t^{2} \partial_{t}+t x \partial_{x}-\left(t u+\frac{1}{2} x\right) \partial_{u}
\end{gathered}
$$

From the commutation relations of these vector fields, we see that $\mathcal{L}$ is a semidirect sum of $\mathfrak{s l}(2, \mathbb{R})$ and an abelian Lie algebra:

$$
\mathcal{L} \sim\left\{P_{0}, D, R\right\} \dot{+}\left\{P_{1}, B\right\} .
$$

As an example let us look at the reductions of the continuous Burgers equation by time translations $P_{0}$. Eq. (4.1) in this case is simply $u_{\lambda}=u_{t}=0$. The Burgers equation (1.1) reduces to the ordinary differential equation

$$
u_{x x}+2 u u_{x}=0 .
$$

We obtain three types of solutions:

$$
u=\frac{1}{x}, \quad u=k \operatorname{arctanh}(k x), \quad u=k \arctan (k x)
$$

depending on whether the first integral of the reduced equation is zero, positive or negative.

The normalizer of $P_{0}$ in the invariance algebra is nor $\left\{P_{0}\right\}=\left\{D, P_{1}, P_{0}\right\}$, so we can use either $D$, or $P_{1}$, to perform a further reduction of eq. (4.5). A reduction by $P_{1}$ leads to the trivial solution $u=$ $u_{0}=$ const. Invariance under dilations provides the first of the three solutions in eq. (4.6). 


\subsection{Symmetry reduction for the discrete Burgers equation}

We have shown that all the symmetries of the discrete Burgers equation (2.6) can be written in the form (3.14) with $\mathcal{S} \phi / \phi$ expressed in terms of $u$. This allows us to write all the reduction formulas in the form $\Delta_{x}(\mathcal{S} \phi / \phi)=0$. Hence, we can in all cases integrate once and write the reduction equations, (i.e. the surface condition) as

$$
\frac{\mathcal{S} \phi}{\phi}=K(t)
$$

and then rewrite eq. (4.7) in terms of $u$. In general $\mathcal{S}$ is a linear combination of all the symmetry operators for the heat equation, i.e. the operators on the right hand sides of eq. (3.1), .., (3.9). Instead of performing a general subalgebra analysis [22], we shall just look at the individual basis elements of the Lie algebra.

\subsubsection{Time translations}

We rewrite $(3.22)$ as

$$
u_{\lambda_{1}}=\left(1+\sigma_{x} u\right) \Delta_{x}\left(\frac{\Delta_{t} \phi}{\phi}\right)
$$

Eq. (4.7) then is

$$
\Delta_{t} \phi=K(t) \phi
$$

or in terms of $u$ :

$$
v=\Delta_{x} u+u T_{x} u=K(t) .
$$

The Burgers equation (2.6) can be written as

$$
\Delta_{t} u=\frac{1+\sigma_{x} u}{1+\sigma_{t} v} \Delta_{x} v
$$

Hence, in view of eq. (4.10) we have

$$
\Delta_{t} u=0, \quad K=K_{0}=\text { const. }
$$

Since $\phi$ satisfies the heat equation we rewrite eq. (4.9) as

$$
\Delta_{x x} \phi=K \phi .
$$

This is a linear difference equation with constant coefficients, and we can easily solve it, putting $\phi=$ $a^{x}$ and finding $a$.

For $K \neq 0$ the general solution of eq. (4.13) is

$$
\phi=c_{1}\left(1+\sqrt{K} \sigma_{x}\right)^{x / \sigma_{x}}+c_{2}\left(1-\sqrt{K} \sigma_{x}\right)^{x / \sigma_{x}},
$$

where $c_{1}$ and $c_{2}$ are arbitrary real constants for $K>0$ and are complex, satisfying $c_{2}=\overline{c_{1}}$ for $K<0$.

For $K=0$ the solution of eq. (4.13) is

$$
\phi=c_{1}+c_{2} x, \quad c_{1}, c_{2} \in \mathbb{R} .
$$

In all cases the corresponding invariant solution of the discrete Burgers equation is obtained via the Cole-Hopf transformation as $u=\Delta_{x} \phi / \phi$. In particular eq. (4.15) yields a solutions invariant under dilations that can be written as

$$
u=\frac{1}{x+\mu \sigma_{x}}, \quad \mu=\text { const. }
$$




\subsubsection{Space translations}

In this case the result is trivial. From eq. (3.23) we have directly $\Delta_{x} u=0$, from the discrete Burgers equation $\Delta_{t} u=0$ and hence $u=$ const.

\subsubsection{Galilei invariance}

Substituting $\mathcal{S}$ from eq. (3.6) into (4.7) we obtain the linearized reduced equation

$$
2\left(t+\sigma_{t}\right) \Delta_{x} \phi+\left(x+\frac{1}{2} \sigma_{x}\right) T_{x}^{-1} \phi+\sigma_{t}\left(x+\frac{1}{2} \sigma_{x}\right) T_{x}^{-1} \Delta_{x x} \phi \quad=K(t)\left(\phi+\sigma_{t} \Delta_{x x} \phi\right) .
$$

In terms of $u$ the reduced equation is obtained from eq. (3.20) and is

$$
\begin{aligned}
& 2 t T_{x} u+x-K(t)+2 t \sigma_{x} u T_{x} u+\sigma_{t}\left(\frac{7}{2} T_{x} u+\frac{7}{2} \sigma_{x} u T_{x} u+x u T_{x} u\right. \\
& \left.+x \Delta_{x} u-\frac{3}{2} u\right)+\frac{3}{2} \sigma_{x}-K(t)\left[\sigma_{x} u+\sigma_{t}\left(T_{x} \Delta_{x} u+u T_{x}^{2} u-u T_{x} u\right)\right. \\
& \left.+T_{x} u T_{x}^{2} u+\sigma_{x} u T_{x} u T_{x}^{2} u\right]=0 .
\end{aligned}
$$

These are difference equations in one variable (namely $x$ ) only; $t$ is a parameter. Eq. (4.17) is a linear second order equation, but it has variable coefficients. Solving it is a nontrivial task which we shall not attempt here.

The situation is similar for the dilations (3.7) and (3.25) and the projective transformation corresponding to eq. (3.8) and (3.26). We shall just present the reduced equations for $\phi$ and $u$, the first being a linearization (via the discrete Cole-Hopf transformation) of the second.

\subsubsection{Scaling invariance}

The linearized reduced equation is

$$
\begin{aligned}
2\left(t+\sigma_{t}\right) \Delta_{x x} \phi+x T_{x}^{-1}\left(\Delta_{x} \phi+\sigma_{t} \Delta_{x x x} \phi\right) & \\
& +\left(1-\frac{1}{2} T_{x}^{-1}\right)\left(\phi+\sigma_{t} \Delta_{x x} \phi\right)=K(t)\left[\phi+\sigma_{t} \Delta_{x x} \phi\right] .
\end{aligned}
$$

The reduced equation for $u$ is:

$$
\begin{array}{r}
\frac{1}{2}+K(t)-u\left[x+\sigma_{x}(2-K(t))-v_{1}\left(2 t-\frac{3}{2} \sigma_{t}\right)\right] \\
-u\left(T_{x} v_{1}\right)\left[2 t+4 \sigma_{x} \sigma_{t}-x \sigma_{t}-K(t) \sigma_{x} \sigma_{t}\right] \\
\quad-\left(\Delta_{x} v_{1}\right)\left(2 t-x \sigma_{t}\right)-\sigma_{t}(u-K(t)) T_{x} v_{1}=0 .
\end{array}
$$

We have defined

$$
v_{j+1}=\Delta_{x} v_{j}+u T_{x} v_{j}, \quad v_{0}=u, \quad v_{1}=v .
$$

\subsubsection{Projective invariance}

The linearized reduced equation for $\phi$ and the reduced equation for $u$ are respectively

$$
\begin{array}{r}
\left(t+2 \sigma_{t}\right)^{2} \Delta_{x x} \phi+\left(t+2 \sigma_{t}\right) x \\
+\left(\frac{1}{4} x_{x}^{-1}\left(\Delta_{x} \phi+\frac{1}{16} \sigma_{x}^{2}\right) T_{x}^{-2} \Delta_{x x x} \phi\right) \\
+\left(t+2 \sigma_{t}\right)\left[\phi-\frac{1}{2} T_{x}^{-1}\left(\phi+\sigma_{t} \Delta_{x x} \phi+\sigma_{t}^{2} \Delta_{x x x x} \phi\right)\right] \\
=K(t)\left(\phi+2 \sigma_{t} \Delta_{x x} \phi+\sigma_{t}^{2} \Delta_{x x x x} \phi\right)
\end{array}
$$


and

$$
\begin{aligned}
\left(t+2 \sigma_{t}\right)^{2}\left(v_{1}+2 \sigma_{x} v_{2}+\right. & \left.\sigma_{x}^{2} v_{3}\right) \\
& +\left(t+2 \sigma_{t}\right)\left(x+\sigma_{x}\right)\left(u+\sigma_{x} v_{1}+\sigma_{t} v_{2}+\sigma_{x} \sigma_{t} v_{3}\right) \\
& \quad+\frac{1}{4}\left[\left(x+2 \sigma_{x}\right)^{2}-\frac{1}{4} \sigma_{x}^{2}\right]\left(1+2 \sigma_{t} v_{1}+\sigma_{t}^{2} v_{3}\right) \\
& +\left(t+2 \sigma_{t}\right)\left(\frac{1}{2}+\frac{3}{2} \sigma_{x} u+\sigma_{x}^{2} v_{1}-\frac{1}{2} \sigma_{t} v_{1}-\frac{1}{2} \sigma_{x} \sigma_{t} v_{2}\right) \\
= & K(t)\left(1+2 \sigma_{x} u+\sigma_{x}^{2} v_{1}+2 \sigma_{t} v_{1}+4 \sigma_{x} \sigma_{t} v_{2}+2 \sigma_{t} \sigma_{x}^{2} v_{3}\right. \\
& \left.+\sigma_{t}^{2} v_{3}+2 \sigma_{t}^{2} \sigma_{x} v_{4}+\sigma_{t}^{2} \sigma_{x}^{2} v_{5}\right)
\end{aligned}
$$

\section{Conclusions}

We have seen on the example of the discrete Burgers equation that conceptually the treatment of symmetries of difference equations is quite similar to the situation for differential ones. Thus, one can construct a Lie algebra of flows commuting with the original equation and write down the corresponding evolutionary vector fields. Subalgebras of the symmetry algebra can be put to good use. As we have shown, they provide reductions to difference equations with fewer independent variables, in our example to ordinary difference equations. There are however important technical differences. Some points that we wish to make are:

1. The symmetries that have Lie point symmetries as their continuous limits are not necessarily point ones in the discrete case. Indeed, all the symmetries $(3.4), \ldots,(3.8)$ of the discrete heat equation involve the function $\phi(x, t)$ at more than one point of the lattice. The only exception is the symmetry (3.9). Similarly, none of the symmetries $(3.22), \ldots,(3.26)$ of the discrete Burgers equation involves $u(x, t)$ only (see eq. (2.2)).

2. We greatly profited from the linearizability of the nonlinear Burgers equation. Indeed the symmetries (3.22), . . , (3.26) were obtained using the discrete Cole-Hopf transformation, applied to the heat equation and its symmetries. In principle these symmetries could be obtained directly using the prolongation techniques introduced in an earlier article [4]. However, the general form (3.1) of the vector field must be used. The resulting formulas (3.22), . .,(3.26) show that the determining equations are not easy to solve (even though they are linear difference equations).

3. All symmetries $(3.22), \ldots,(3.26)$ involve shifts to a finite (and small) number of neighboring points on the lattice. The symmetries (3.28) and (3.29) involve infinitely many points (and end up being ordinary continuous derivatives in one point).

4. Each symmetry provides a reduction of the original equation to an ordinary difference equation. The invariance conditions are however in general nonlinear higher order difference equations. They may be quite difficult to solve, even though they are (for the Burgers equation) always linearizable.

5. A "naive" discretization of the Burgers equation would lead to the equation

$$
\Delta_{t} u=\Delta_{x x} u+2 u \Delta_{x} u
$$

rather than to equation (2.6). The naive discretization loses all the essential properties of the Burgers equation: its linearizability and its symmetries. More generally, discretization 
procedures are not unique and different linearizations may be preferable, depending on what we want to preserve (linearizability, integrability, symmetries, conservation laws, etc.).

In short, Lie symmetry techniques are useful for difference equations, but the distinction between point and higher symmetries becomes blurred. All the complications that arise in the study of higher symmetries of differential equations must be addressed in the case of difference ones.

\section{Acknowledgments}

We thank Luc Vinet for helpful discussions. Research grants from NSERC of Canada, FCAR du Quebec, MURST of Italy, CICYT PB95-0401 from Spain and NATO (CRG 960717) are gratefully acknowledged.

\section{References}

[1] J. M. Burgers, The Nonlinear Diffusion Equation: Asymptotic Solutions and Statistical Problems, Reidel, Dordrecht, 1974.

[2] P. J. Olver, Applications of Lie Groups to Differential Equations, Springer, Berlin, 1986.

[3] R. Floreanini and L. Vinet, Lie Symmetries of Finite Difference Equations, J. Math. Phys. 36, 7024-7042 (1995).

[4] D. Levi, L. Vinet and P. Winternitz, Lie Group Formalism for Difference Equations, J. Phys. A: Math. Gen. 30, 633-649 (1997).

[5] D. Levi and P. Winternitz, Continuous Symmetries of Discrete Equations, Phys. Lett. A 152, 335-338 (1991)

[6] D. Levi and P. Winternitz, Symmetries and Conditional Symmetries of Differential-Difference Equations, J. Math. Phys. 34, 3713-3730 (1993).

[7] D. Levi and P. Winternitz, Symmetries of Discrete Dynamical Systems, J. Math. Phys. 37, 5551-5576 (1996).

[8] S. Lafortune, L. Martina and P. Winternitz, Symmetries of Toda Field Theories and their Reductions, Preprint CRM 2566, Montréal, 1998.

[9] D. Gómez Ullate, S. Lafortune and P. Winternitz, Symmetries of Discrete Dynamical Systems Involving Two Species, Preprint CRM 2567, Montréal, 1998.

[10] R. Hernández Heredero, D. Levi and P. Winternitz, Point Symmetries and Generalized Symmetries of Nonlinear Difference Equations, Preprint CRM, Montréal, 1998.

[11] D. Levi and M. A. Rodríguez, Symmetry Groups of Partial Differential Equations and of Differential-Difference Equations. The Toda Lattice versus the Korteweg-de Vries Equation. J. Phys. A: Math. Gen. 25, L875-L979 (1992).

[12] D. Levi and R. Yamilov, Conditions for the Existence of Higher Symmetries of Evolutionary Equations on a Lattice, J. Math. Phys. 38, 6648-6674 (1997). 
[13] D. Levi, L. Vinet and P. Winternitz (Editors). Symmetries and Integrability of Difference Equations, CRM Proceedings and Lecture Notes, Vol. 9, AMS, Providence, R.I., 1996.

[14] G. R. W. Quispel, H. W. Capel and R. Sahadevan, Continuous Symmetries of DifferentialDifference Equations: the Kac-van Moerbeke Equation and Painlevé Reduction. Phys. Lett. A 170 379-383 (1992).

[15] V. Dorodnitsyn, Transformation Groups in Mesh Spaces, J. Soviet Math. 55, 1490-1517 (1991).

[16] N. Joshi and P. J. Vassiliou, The Existence of Lie Symmetries for First Order Analytic Discrete Dynamical Systems, J. Math. Anal. Appl. 195, 872-887 (1995).

[17] S. Maeda, The Similarity Method for Difference Equations, IMA J. Appl. Math. 38, 129 (1987).

[18] Zhuhan Jiang, Lie Symmetries and Their Local Determinancy for a Class of DifferentialDifference Equations, Phys. Lett. A240, 137-143 (1998).

[19] R. Hirota, J. Phys. Soc. Japan 46, 312 (1979).

[20] K. Nishinari and D. Takahashi, Analytical properties of ultradiscrete Burgers equation and rule-184 cellular automaton, J. Phys. A: Math. Gen. 31, 5439-5450 (1998).

[21] D. Levi, O. Ragnisco and M. Bruschi, Continuous and Discrete Matrix Burgers Hierarchies, Nuovo Cim. B11, 74, 33-51 (1993)

[22] P. Winternitz, Lie Groups and Solutions of Nonlinear Partial Differential Equations. In Integrable Systems, Quantum Groups and Quantum Field Theories, p. 429-495, Kluwer, Dordrecht, 1993, Editors A. Ibort and M.A. Rodriguez. 